\title{
Leading-Order Behavior of the Correlation Energy in the Uniform Electron Gas
}

\author{
PIERRE-FRANÇOIS LOOS, PETER M. W. GILL \\ Research School of Chemistry, Australian National University, Canberra, ACT 0200, Australia
}

Received 31 March 2011; accepted 22 April 2011

Published online 24 June 2011 in Wiley Online Library (wileyonlinelibrary.com).

DOI 10.1002/qua.23155

\begin{abstract}
We show that, in the high-density limit, restricted Møller-Plesset (RMP) perturbation theory yields $E_{\mathrm{RMP}}^{(2)}=\pi^{-2}(1-\ln 2) \ln r_{\mathrm{s}}+O\left(r_{\mathrm{s}}^{0}\right)$ for the correlation energy per electron in the uniform electron gas, where $r_{\mathrm{s}}$ is the Seitz radius. This contradicts an earlier derivation which yielded $E_{\mathrm{RMP}}^{(2)}=O\left(\ln \left|\ln r_{\mathrm{s}}\right|\right)$. The reason for the discrepancy is explained. (ㅇ 2011 Wiley Periodicals, Inc. Int J Quantum Chem 112: 1712-1716, 2012
\end{abstract}

Key words: jellium; uniform electron gas; Hartree-Fock; perturbation theory

W e consider a paramagnetic system of $N$ interacting electrons confined in a cubic box, with edges of length $L$ and volume $\Omega=L^{3}$. We also assume a uniform charge density background of density equal in magnitude, but opposite in sign, to the average electron density $\rho=N / \Omega$. In the thermodynamic limit, both $N$ and $\Omega$ tend to infinity in such a way that the system becomes homogeneous with a uniform density $\rho$, related to the Seitz radius by the relation $r_{\mathrm{s}}=(4 \pi \rho / 3)^{-1 / 3}$ and is often called jellium [1, 2].

It is convenient to consider a reduced Hamiltonian (i.e., one that is scaled by the number of electrons) and, in atomic units, this is

Correspondence to: P. M. W. Gill; e-mail: peter.gill@anu.edu.au Contract grant sponsor: Australian Research Council. Contract grant number: DP0984806, DP1094170.

$$
\hat{H}=N^{-1}\left[\hat{T}+\hat{H}_{\mathrm{e}-\mathrm{e}}+\hat{H}_{\mathrm{e}-\mathrm{b}}+\hat{H}_{\mathrm{b}-\mathrm{b}}\right],
$$

where the operator

$$
\hat{T}=-\frac{1}{2} \sum_{i=1}^{N} \nabla_{i}^{2}
$$

corresponds to the kinetic energy of the electrons, and

$$
\begin{aligned}
& \hat{H}_{\mathrm{e}-\mathrm{e}}=\sum_{i<j}^{N} \frac{1}{\left|\boldsymbol{r}_{i}-\boldsymbol{r}_{j}\right|}, \\
& \hat{H}_{\mathrm{e}-\mathrm{b}}=-\rho \sum_{i=1}^{N} \int \frac{d \boldsymbol{R}}{\left|\boldsymbol{r}_{i}-\boldsymbol{R}\right|}, \\
& \hat{H}_{\mathrm{b}-\mathrm{b}}=\frac{\rho^{2}}{2} \iint \frac{d \boldsymbol{R}_{1} d \boldsymbol{R}_{2}}{\left|\boldsymbol{R}_{1}-\boldsymbol{R}_{2}\right|}
\end{aligned}
$$


represent the electron-electron, electron-background, and background-background interactions, respectively. In the thermodynamic limit, both $\hat{H}_{\mathrm{b}-\mathrm{b}}$ and $\hat{H}_{\mathrm{e}-\mathrm{b}}$ diverge. However, the divergence is cancelled by a term from $\hat{H}_{\mathrm{e}-\mathrm{e}}$. See Ref. [1] for more details. The term $\hat{H}_{\mathrm{b}-\mathrm{b}}$ is a known constant [3] and may be ignored. tion

In perturbation theory [4], we introduce a parti-

$$
\hat{H}=\hat{H}^{(0)}+\hat{V},
$$

where the perturbation $\hat{V}$ is assumed small (in some sense) compared with the zeroth-order Hamiltonian $\hat{H}^{(0)}$. This yields an expansion of the (reduced) energy

$$
E=E^{(0)}+E^{(1)}+E^{(2)}+\ldots .
$$

The zeroth-, first-, and second-order energies are given by

$$
\begin{aligned}
E^{(0, \ell)} & =\left\langle\Psi^{(0, \ell)}\left|\hat{H}^{(0)}\right| \Psi^{(0, \ell)}\right\rangle, \\
E^{(1)} & =\left\langle\Psi^{(0)}|\hat{V}| \Psi^{(0)}\right\rangle, \\
E^{(2)} & =\sum_{\ell=1}^{\infty} \frac{\left\langle\Psi^{(0, \ell)}|\hat{V}| \Psi^{(0)}\right\rangle^{2}}{E^{(0, \ell)}-E^{(0)}},
\end{aligned}
$$

where $E^{(0,0)} \equiv E^{(0)}$ and $\Psi^{(0,0)} \equiv \Psi^{(0)}$, and the zerothorder ground state $(\ell=0)$ and excited states $(\ell>0)$ wave functions satisfy

$$
\hat{H}^{(0)} \Psi^{(0, \ell)}=E^{(0, \ell)} \Psi^{(0, \ell)} .
$$

There are many ways to partition $\hat{H}$ but not all are equally effective. In this article, we will consider three: the noninteracting (NI), restricted MøllerPlesset [5] (RMP), and unrestricted Møller-Plesset (UMP) partitions.

If we adopt the NI partition, we have

$$
\hat{H}_{\mathrm{NI}}^{(0)}=\hat{T}, \quad \hat{V}_{\mathrm{NI}}=\hat{H}_{\mathrm{e}-\mathrm{e}}+\hat{H}_{\mathrm{e}-\mathrm{b}} .
$$

The zeroth-order wave functions $\Psi_{\mathrm{NI}}^{(0, \ell)}$ are Slater determinants of plane-wave orbitals

$$
\psi_{k}(\boldsymbol{r})=\frac{1}{\Omega} \exp (i \boldsymbol{k} \cdot \boldsymbol{r})
$$

with orbital energies

$$
\epsilon_{\mathrm{NI}}(k)=\frac{k^{2}}{2} .
$$

The $\ell$ th excited determinant $\Psi_{\mathrm{NI}}^{(0, \ell)}$ has the energy

$$
E_{\mathrm{NI}}^{(0, \ell)}=\frac{1}{N} \sum_{k}^{\mathrm{occ}} \epsilon_{\mathrm{NI}}(k),
$$

where the sum over $k$ takes into account all the plane waves used to build $\Psi_{\mathrm{NI}}^{(0, \ell)}$, i.e., all the occupied orbitals in the state $\ell$. For the special case $\ell=0$, all the orbitals up to the Fermi level are occupied.

Introducing $\alpha=(9 \pi / 4)^{1 / 3}$, one finds [6-8] that

$$
E_{\mathrm{NI}}^{(0)}=\frac{3}{10} \frac{\alpha^{2}}{r_{\mathrm{s}}^{2}}, \quad E_{\mathrm{NI}}^{(1)}=-\frac{3}{4 \pi} \frac{\alpha}{r_{\mathrm{s}}},
$$

which are the kinetic and exchange energies, respectively. The Coulomb part in $E_{\mathrm{NI}}^{(1)}$ is exactly cancelled by the positive uniform background via the term $\hat{H}_{\mathrm{e}-\mathrm{b}}$.

Unfortunately, although the correlation energy [9]

$$
E_{\mathrm{c}}=E-E^{(0)}-E^{(1)}
$$

of jellium is known [10] to be finite for any $r_{\mathrm{s}}>0$, the second-order energy Eq. (10) is infinite. However, the leading-order contribution can be extracted from Eq. (10) and, henceforth, we will use $E^{(2)}$ to refer to that contribution.

After transforming into momentum space and scaling the momenta by the wave vector $k_{\mathrm{F}}=\alpha / r_{\mathrm{s}}$ so that the Fermi sphere has unit radius, one finds $[11,12]$ that $E_{\mathrm{NI}}^{(2)}$ consists of a direct ("ring-diagram") contribution

$$
E_{\mathrm{NI}}^{(2, \mathrm{a})}=-\frac{3}{16 \pi^{5}} \iiint \frac{d \boldsymbol{q} d \boldsymbol{k}_{1} d \boldsymbol{k}_{2}}{q^{4} \Delta \epsilon_{\mathrm{NI}}},
$$

and an exchange contribution

$$
E_{\mathrm{NI}}^{(2, \mathrm{~b})}=\frac{3}{32 \pi^{5}} \iiint \frac{d \boldsymbol{q} d \boldsymbol{k}_{1} d \boldsymbol{k}_{2}}{\boldsymbol{q}^{2}\left|\boldsymbol{q}+\boldsymbol{k}_{1}-\boldsymbol{k}_{2}\right|^{2} \Delta \epsilon_{\mathrm{NI}}} .
$$

In these integrals, the excitation vector $q$ has the domain

$$
\beta<|q|<\infty,
$$

where $\beta \propto \sqrt{r_{\mathrm{s}}}$ [13], and the occupied-orbital vectors $k_{1}$ and $k_{2}$ have domains

$$
\begin{array}{ll}
\left|k_{1}\right|<1, & \left|k_{1}+q\right|>1, \\
\left|k_{2}\right|<1, & \left|k_{2}-q\right|>1,
\end{array}
$$


The lower bound for $q$ in Eq. (20) is due to the screening effect of the Coulomb field by the collective electron motions, and can be derived using the plasma theory of the free-electron gas [13-17]. The orbital energy difference is

$\Delta \epsilon_{\mathrm{NI}}$

$$
=\epsilon_{\mathrm{NI}}\left(\left|\boldsymbol{k}_{1}+\boldsymbol{q}\right|\right)+\epsilon_{\mathrm{NI}}\left(\left|\boldsymbol{k}_{2}-\boldsymbol{q}\right|\right)-\epsilon_{\mathrm{NI}}\left(k_{1}\right)-\epsilon_{\mathrm{NI}}\left(k_{2}\right) .
$$

The exchange term $E_{\mathrm{NI}}^{(2, \mathrm{~b})}$ is finite [18] and, for small $r_{\mathrm{s}}$, is dominated by the ring-diagram term

$$
E_{\mathrm{NI}}^{(2, \mathrm{a})}=\frac{1-\ln 2}{\pi^{2}} \ln r_{\mathrm{s}}+O\left(r_{\mathrm{s}}^{0}\right),
$$

which Macke showed [11] to depend logarithmically on $r_{\mathrm{s}}$. One may wonder, however, whether this logarithmic term arises when the Hamiltonian is partitioned differently [19].

If we adopt the RMP partition [20], we have

$$
\hat{H}_{\mathrm{RMP}}^{(0)}=\sum_{i=1}^{N} \hat{F}\left(\boldsymbol{r}_{i}\right), \quad \hat{V}_{\mathrm{RMP}}=\hat{H}-\sum_{i=1}^{N} \hat{F}\left(\boldsymbol{r}_{i}\right),
$$

where the Fock operator defined by

$$
\begin{aligned}
\hat{F}\left(\boldsymbol{r}_{1}\right) \psi_{\boldsymbol{k}_{1}}\left(\boldsymbol{r}_{1}\right) & \\
= & =\frac{1}{2} \nabla_{1}^{2} \psi_{k_{1}}\left(\boldsymbol{r}_{1}\right)+\sum_{k_{2}}^{\mathrm{occ}} \psi_{k_{2}}\left(\boldsymbol{r}_{1}\right) \int \frac{\psi_{k_{2}}^{*}\left(\boldsymbol{r}_{2}\right) \psi_{k_{1}}\left(\boldsymbol{r}_{2}\right)}{\left|\boldsymbol{r}_{1}-\boldsymbol{r}_{2}\right|} d \boldsymbol{r}_{2}
\end{aligned}
$$

includes kinetic and exchange terms but not Hartree terms because of their cancelation by the $\hat{H}_{\mathrm{e}-\mathrm{b}}$ term.

The RMP zeroth-order wave functions $\Psi_{\mathrm{RMP}}^{(0, \ell)}$ are again determinants of plane-wave orbitals (13), but the orbital energies are now different and it can be shown $[21,22]$ that

$$
\epsilon_{\mathrm{RMP}}(k)=\epsilon_{\mathrm{NI}}(k)-\frac{r_{\mathrm{s}}}{\alpha \pi} \epsilon_{\mathrm{X}}(k) .
$$

The additional term

$$
\epsilon_{\mathrm{X}}(k)=1+\frac{1-k^{2}}{2 k} \ln \left|\frac{1+k}{1-k}\right|
$$

arises from the exchange terms in Eq. (26). Thus,

$$
\Psi_{\mathrm{RMP}}^{(0, \ell)}=\Psi_{\mathrm{NI}}^{(0, \ell)},
$$

but

$$
E_{\mathrm{RMP}}^{(0, \ell)}=\frac{1}{N} \sum_{k}^{\mathrm{occ}} \epsilon_{\mathrm{RMP}}(k) \neq E_{\mathrm{NI}}^{(0, \ell)} .
$$

The zeroth- and first-order energies are now given by

$$
E_{\mathrm{RMP}}^{(0)}=\frac{3}{10} \frac{\alpha^{2}}{r_{\mathrm{s}}^{2}}-\frac{3}{2 \pi} \frac{\alpha}{r_{\mathrm{s}}}, \quad E_{\mathrm{RMP}}^{(1)}=\frac{3}{4 \pi} \frac{\alpha}{r_{\mathrm{s}}},
$$

and comparing Eqs. (16) and (31) reveals the important relation

$$
E_{\mathrm{NI}}^{(0)}+E_{\mathrm{NI}}^{(1)}=E_{\mathrm{RMP}}^{(0)}+E_{\mathrm{RMP}}^{(1)}=E_{\mathrm{RHF}},
$$

where $E_{\mathrm{RHF}}$ is the reduced RHF energy.

The ring-diagram contribution to $E_{\mathrm{RMP}}^{(2)}$ is

$$
E_{\mathrm{RMP}}^{(2, \mathrm{a})}=-\frac{3}{16 \pi^{5}} \iiint \frac{d \boldsymbol{q} d \boldsymbol{k}_{1} d \boldsymbol{k}_{2}}{q^{4} \Delta \epsilon_{\mathrm{RMP}}}
$$

which differs from Eq. (18) only by the denominator

$$
\Delta \epsilon_{\mathrm{RMP}}=\Delta \epsilon_{\mathrm{NI}}-\frac{r_{\mathrm{s}}}{\alpha \pi} \Delta \epsilon_{\mathrm{X}}
$$

where

$$
\Delta \epsilon_{\mathrm{X}}=\epsilon_{\mathrm{X}}\left(\left|\boldsymbol{k}_{1}+\boldsymbol{q}\right|\right)+\epsilon_{\mathrm{X}}\left(\left|\boldsymbol{k}_{2}-\boldsymbol{q}\right|\right)-\epsilon_{\mathrm{X}}\left(k_{1}\right)-\epsilon_{\mathrm{X}}\left(k_{2}\right) .
$$

The behavior of $E_{\mathrm{RMP}}^{(2, \mathrm{a})}$ is dominated [22] by contributions in the neighborhood of the Fermi sphere (i.e., $q \approx 0$ ). On the domains (21) and (22), we have

$$
\epsilon_{\mathrm{RMP}}(k)=\frac{k^{2}}{2}-\frac{r_{\mathrm{s}}}{\alpha \pi}\left[1+\frac{1-k^{2}}{2 k} \ln \frac{1+k}{1-k}\right],
$$

and

$$
\begin{aligned}
\epsilon_{\mathrm{RMP}}(|\boldsymbol{k}+\boldsymbol{q}|) & =\frac{|\boldsymbol{k}+\boldsymbol{q}|^{2}}{2} \\
& -\frac{r_{\mathrm{s}}}{\alpha \pi}\left[1+\frac{1-|k+\boldsymbol{q}|^{2}}{2|\boldsymbol{k}+\boldsymbol{q}|} \ln \frac{|\boldsymbol{k}+\boldsymbol{q}|+1}{|\boldsymbol{k}+\boldsymbol{q}|-1}\right] .
\end{aligned}
$$

Therefore, we have

$$
\Delta \epsilon_{\mathrm{RMP}} \approx u+v-\frac{r_{\mathrm{s}}}{\alpha \pi}\left(u \ln \frac{u}{2}+v \ln \frac{v}{2}\right),
$$

where we have introduced

$$
u=\frac{k_{1} \cdot \boldsymbol{q}}{k_{1}}, \quad v=-\frac{k_{2} \cdot \boldsymbol{q}}{k_{2}} .
$$


Substituting (38) into (33) and using the relations

$$
\begin{gathered}
d \boldsymbol{k}_{1}=2 \pi k_{1}^{2} \sin \theta d \theta d k_{1} \approx \frac{2 \pi}{q} d u d k_{1}, \\
\left|\boldsymbol{k}_{1}+\boldsymbol{q}\right|>1 \Rightarrow 1-u \leq k_{1} \leq 1,
\end{gathered}
$$

(with similar expressions for $k_{2}$ ) then yields

$$
\begin{aligned}
E_{\mathrm{RMP}}^{(2, \mathrm{a})} \approx & -\frac{3}{\pi^{2}} \int_{\beta}^{1} \frac{d q}{q^{4}} \int_{0}^{q} d u \int_{1-u}^{1} d k_{1} \int_{0}^{q} d v \\
& \times \int_{1-v}^{1} d k_{2} \frac{1}{u+v-\frac{r_{\mathrm{s}}}{\alpha \pi}\left(u \ln \frac{u}{2}+v \ln \frac{v}{2}\right)} \\
= & -\frac{3}{\pi^{2}} \int_{\beta}^{1} \frac{d q}{q^{4}} \int_{0}^{q} d u \\
& \times \int_{0}^{q} d v \frac{u v}{u+v-\frac{r_{\mathrm{s}}}{\alpha \pi}\left(u \ln \frac{u}{2}+v \ln \frac{v}{2}\right)} .
\end{aligned}
$$

As the most important contribution comes from small $q$, we have set the upper bound of the integral (42) to a convenient value of unity. Expanding for small $r_{\mathrm{s}}$ and integrating over $q$ yields

$$
\begin{aligned}
E_{\mathrm{RMP}}^{(2, \mathrm{a})} \approx & -\frac{3}{\pi^{2}} \int_{\beta}^{1} \frac{d q}{q^{4}} \int_{0}^{q} d u \\
& \times \int_{0}^{q} d v \frac{u v}{u+v}\left[1+\frac{r_{\mathrm{s}}}{\alpha \pi} \frac{u \ln \frac{u}{2}+v \ln \frac{v}{2}}{u+v}\right] \\
= & \frac{1-\ln 2}{\pi^{2}} \ln r_{\mathrm{s}}+O\left(r_{\mathrm{s}}^{0}\right)
\end{aligned}
$$

which is identical, in the high-density (i.e., small$\left.r_{\mathrm{s}}\right)$ limit, to $E_{\mathrm{NI}}^{(2, a)}$. The present result can also be obtained from (42) by first switching to polar coordinates $(u=r \cos \theta$ and $v=r \sin \theta)$, integrating over the radial part, carefully taking the $r_{\mathrm{s}} \rightarrow 0$ limit, and finally performing the remaining angular integration. The latter derivation rigorously justifies the small- $r_{s}$ expansion. We thank the referee for providing this alternative derivation.

In a similar investigation more than 20 years ago [19], Handler claimed to show that

$$
E_{\mathrm{RMP}}^{(2, \mathrm{a})}=O\left(\ln \left|\ln r_{\mathrm{s}}\right|\right),
$$

this claim, which implies that $E_{\mathrm{RMP}}^{(2)}$ grows more slowly with $r_{\mathrm{s}}$ than $E_{\mathrm{NI}}^{(2)}$, obviously disagrees with our result in Eq. (43). However, in his analog of Eq. (42), Handler drops the $u+v$ term and ignores the $r_{s} / \alpha \pi$ factor. Although it does not seem to alter Handler's derivation, the expressions reported in Ref. [19] for
$\epsilon_{\mathrm{RMP}}\left(\left|\boldsymbol{k}_{1}+\boldsymbol{q}\right|\right)$ and $\epsilon_{\mathrm{RMP}}\left(k_{1}\right)$ are incorrect. The fact that $\beta \propto \sqrt{r_{\mathrm{s}}}$ means that Handler's neglect of the $u+v$ term is incorrect.

It may be surprising that $E_{\mathrm{RMP}}^{(2)}$ is the same as $E_{\mathrm{NI}}^{(2)}$, because $\hat{H}_{\mathrm{RMP}}^{(0)}$ seems a better starting point than $\hat{H}_{\mathrm{NI}}^{(0)}$. However, this is not the first time that the RHF treatment of jellium has been disappointing. For example, the RHF bandwidth, $\epsilon(1)-\epsilon(0)$, is greater than the NI bandwidth, which disagrees with experiments on simple metals, where a small reduction is observed $[23,24]$. Moreover, the logarithmic dependence of the eigenvalues (27) leads to a divergent derivative of $\epsilon_{\mathrm{RMP}}(k)$ at the surface of the Fermi sphere $(k=1)$ and this leads to incorrect dependence of the electronic specific heat on temperature. Experimentally, a linear dependence with a prefactor close to the NI value is observed $[25,26]$.

One may hope that a different, and superior, perturbation series can be obtained by adopting the UMP partition, that is, by using the UHF wavefunction of jellium as the starting point. After all, as Overhauser showed long ago [27, 28], the RHF solution of jellium is unstable with respect to a lowerenergy UHF solution, for all $r_{\mathrm{s}}$ [29]. However, we expect that there will be serious issues with the convergence of the UMP perturbation series $[30,31]$ and we have not considered this alternative in detail.

In conclusion, we have shown that the correlation energy $E_{\mathrm{RMP}}^{(2)}$ from RMP perturbation theory, i.e., using a RHF starting point, is the same as the $E_{\mathrm{NI}}^{(2)}$ from conventional NI perturbation theory. Although it is nearly impossible to test experimentally this result, this corrects an earlier study which claimed that $E_{\mathrm{RMP}}^{(2)}$ is sublogarithmic.

\section{ACKNOWLEDGMENTS}

The authors thank Joshua Hollett for many valuable discussions, and the referee for his careful analysis of this article. P.M.W.G. thanks the NCI National Facility for a generous grant of supercomputer time.

\section{References}

1. Giuliani, G. F; Vignale, G. Quantum Theory of Electron Liquid; Cambridge University Press: Cambridge, 2005.

2. Parr, R. G.; Yang, W. Density Functional Theory for Atoms and Molecules; Oxford University Press: Oxford, 1989.

3. Ghosh, S.; Gill, P. M. W. J Chem Phys 2005, 122, 154108.

4. Helgaker, T.; Jørgensen, P.; Olsen, J. Molecular ElectronicStructure Theory; Wiley: 2000. 
5. Møller, C.; Plesset, M. S. Phys Rev 1934, 46, 618.

6. Fermi, E. Z Phys 1926, 36, 902.

7. Thomas, L. H. Proc Cam Philos Soc 1927, 23, 542.

8. Dirac, P. A. M. Proc Cam Philos Soc 1930, 26, 376.

9. Wigner, E. Phys Rev 1934, 46, 1002.

10. Ceperley, D. M.; Alder, B. J. Phys Rev Lett 1980, 45, 566.

11. Macke, W. Z Naturforsch A 1950, 5a, 192.

12. Gell-Mann, M.; Brueckner, K. A. Phys Rev 1957, 106, 364.

13. Bohm, D.; Pines, D. Phys Rev 1953, 92, 609.

14. Bohm, D.; Pines, D. Phys Rev 1951, 82, 625.

15. Pines, D.; Bohm, D. Phys Rev 1952, 85, 338.

16. Pines, D. Phys Rev 1953, 92, 626.

17. Nozières, P.; Pines, D. Phys Rev 1958, 111, 442.

18. Onsager, L.; Mittag, L.; Stephen, M. J. Ann Phys 1966, 18,71 .

19. Handler, G. S. Int J Quantum Chem 1988, 33, 173.
20. Szabo, A.; Ostlund, N. S. Modern Quantum Chemistry: Introduction to Advanced Structure Theory; Dover Publications Inc.: Mineola, New York, 1989.

21. Raimes, S. The Wave Mechanics of Electrons in Metals; Amsterdam: North-Holland, 1961.

22. Raimes, S. Many-Electron Theory; Amsterdam: NorthHolland, 1972.

23. Jensen, E.; Plummer, E. W. Phys Rev Lett 1985, 55, 1912.

24. Lyo, I.-W.; Plummer, E. W. Phys Rev Lett 1988, 60, 1558.

25. Bardeen, J. Phys Rev 1936, 49, 653.

26. Bohm, D.; Pines, D. Phys Rev 1950, 80, 903.

27. Overhauser, A. W. Phys Rev Lett 1959, 3, 414.

28. Overhauser, A. W. Phys Rev 1962, 128, 1437.

29. Zhang, S.; Ceperley, D. M. Phys Rev Lett 2008, 100, 236404.

30. Gill, P. M. W.; Radom, L. Chem Phys Lett 1986, 132, 16.

31. Gill, P. M. W.; Pople, J. A.; Radom, L.; Nobes, R. H. J Chem Phys 1988, 89, 7307. 